\title{
Line Filtering for Surgical Tool Localization in 3D Ultrasound Images
}

\author{
Marián Uherč' $1 k^{\mathrm{a}}$, Jan Kybic ${ }^{\mathrm{a}}$, Yue Zhao ${ }^{\mathrm{b}, *}$, Christian Cachard $^{\mathrm{b}}$, Hervé \\ Liebgott $^{\mathrm{b}}$ \\ ${ }^{a}$ Center for Machine Perception, Department of Cybernetics, Faculty of Electrical \\ Engineering, Czech Technical University in Prague, Czech Republic \\ ${ }^{b}$ Université de Lyon, CREATIS; CNRS UMR5220; Inserm U1044; INSA-Lyon; Université \\ Lyon 1, France.
}

\begin{abstract}
We present a method for automatic surgical tool localization in 3D ultrasound images based on line filtering, voxel classification and model fitting. A possible application is to provide assistance for biopsy needle or micro-electrode insertion, or a robotic system performing this insertion. The line filtering method is first used to enhance the contrast of the $3 \mathrm{D}$ ultrasound image, then a classifier is chosen to separate the tool voxels, in order to reduce the number of outliers. The last step is a RANSAC model fitting. Experimental results on several different datasets demonstrate that the failure rate of the proposed method is lower than for preceding methods, with similar localization accuracy, at the expense of a modest increase in computational effort. Even though some of the line filtering methods are a little slower than other methods, its robustness and accuracy are strongly proposed and it is worth to develop a system for clinique applications.
\end{abstract}

Keywords: 3D ultrasound, tool localization, line filtering, biopsy, RANSAC

\section{Introduction}

In this paper, we present a method for enhancement and localization of surgical tools such as needles or electrodes in 3D ultrasound (US) images. Thin tool insertion [1] is a part of many surgical procedures such as biopsy [2], brachytherapy [3], breast cancer therapy and diagnostics [4], or neuron activity recording [5]. A localization system can assist the surgeon in the tool insertion procedure by highlighting the tool position, providing navigation with respect to the target, and choosing the proper plane from the 3D volume for visualization; this will become increasingly important as $3 \mathrm{D}$ ultrasound probes become more widely used. Automatic tool localization is also important for the accuracy and robustness of robotic tool insertion systems.

\footnotetext{
* Corresponding author

Email address: yue.zhao@creatis.insa-lyon.fr (Yue Zhao)
} 


\subsection{Existing tool localization techniques}

First devices to localize and guide surgical tools were mechanical [6]. Here, we will concentrate on techniques based on medical imaging [7], and especially ultrasound [8], because of its speed, cost effectivity and non-invasiveness.

Most tool localization techniques for 3D ultrasound are based on projections and Hough transform or Radon transform variants [9, 10, 11, 12, 13, 14, 15]. One general disadvantage of projection methods is their computational complexity, even though almost real-time speed can be achieved by using multiresolution [16, 17] and Graphics Processing Unit (GPU) implementation [18]. Mari et al [19] have proposed a fast approach which first limits the region of a 3D US volume, then uses a parallel integral projection (PIP) [14] to locate the position of a small straight tool. A more serious problem is that projection methods are easily confused if other highly echogenic objects are present in the image.

In our previous work [20] we have introduced another type of tool localization technique, which first finds candidate voxels which are likely to belong to the tool, and then fits the selected voxels by a parametric model of the tool shape. The model fitting approach is much faster than projection-based methods because only a small fraction of the voxels is considered. It is also more general by not assuming the object to be straight. Robustness is achieved using RANSAC [21] and by using a voxel classifier based on voxel intensity and distance from the assumed tool axis; classifier parameters are learnt from training data. RANSAC is used to find an approximate solution, which is subsequently refined in a local optimization step. We have shown experimentally [20] that the model fitting method achieves the lowest failure rate from all tested method. Nevertheless, the obtained failure rate is still too high to allow clinical applicability; it can approach $100 \%$ on our most challenging datasets (e.g. breast biopsy). In the present work we show how to decrease the failure rate almost to zero.

\subsection{Proposed method}

In this paper, we propose to use the shape information provided by line filtering (see Section 2) to improve the previously described automatic localization method [20], particularly its robustness with respect to large and bright background structures and noise. More specifically, the line filtering is originally used to enhance the structure of blood vessel, we now use the fact that it would be able to enhance one-dimensional (1D) structures such as a needle or an electrode. This should help to suppress incorrect (false positive) detections of other highly echogenic image structures such as locally planar (2D) interfaces with bones or fat, round ball-like (3D) structures or unstructured noise. A secondary benefit of line filtering is the enhancement of visual contrast which aids localization for human observers. There are two hypothesizes in this study: a) the intensity of the needle voxels is higher than the surrounding tissue; b), the shape of the tool is a thin, long, and uncurved cylinder. Bending is typical for thin electrodes (diameter around $0.3 \mathrm{~mm}$ ). Biopsy needles are thicker (diameter around $1 \mathrm{~mm}$ ) and therefore remain straight. 
We review existing line filtering methods in Section 2 and describe our new tool localization algorithm in Section 3; Section 4 contains experimental evaluation. Finally, Section 6 and Section 7 give the existing problem and conclusion respectively.

\section{Line filtering methods}

Line filtering is a method for enhancement of $1 \mathrm{D}$ line structures, originally developed for vessel enhancement and detection [22]. In our case the tool diameter is fixed and known, which makes the task slightly easier.

A matching filter approach applies a set of filters of a similar shape as the object being sought. Chaudhuri et al. [23] use matched filters for detection of blood vessels in $2 \mathrm{D}$ images. 12 various orientations with angular distance $15^{\circ}$ are prepared, and the kernel width $\sigma$ is determined manually. Hoover et al [24] is based on Chaudhuri's method, and he improves it by using the optimized value of $\sigma$. The threshold probing procedure proposed by Hoover et al classifies each pixel using local and region-based properties. The response of the matehed filter is examined by iteratively decreasing the threshold of the classsifier. The accuracy for methods based on matched filter depends on the number of filters, but the more the filters, the lower the calculation speed. The size of the convolution mask also influence the speed.

Compared to matched filters, steerable filters [25], are a computationally efficient approach for evaluating responses for many angles by interpolating between responses of only a small number of basis filters. Freeman et al. [25] has introduced the design of the steerable filter and provides several application examples. Gonzalez et al. [26] use the steerable filter for line detection in the 3D MRA images. They first use the steerable features for statistical 3D dendrite detection. Then a classifier is learned to adapt the filter to particular properties of filaments without manual intervention.

Linear structures can also be seen as ridges in the corresponding higher dimensional space. Aylward et al [27] approximate the medial axes of tubular vessels as oriented intensity ridges. Ridges are tracked from a user-supplied starting point by estimating the local directions of the tube with respect to the Hessian matrix. The local widths of the object is estimated by using points on the ridges. Staal et al. [28] use the Hessian based method to detect ridges in $2 \mathrm{D}$ images followed by a learn-able selection scheme. Ridge pixels are grouped by the similarity of eigenvector directions, and straight line elements are formed out of them. Image is partitioned according to the closest line element. [29] has shown that the the elements of the Hessian matrix also constitute a basis for a steerable filter. In next section, the Hessian based analysis is described. 


\subsection{Hessian based analysis}

Local intensity variations are analyzed via second order derivatives assembled in a Hessian matrix

$$
M(\mathbf{x})=\left[\begin{array}{ccc}
I_{x x}(\mathbf{x}) & I_{x y}(\mathbf{x}) & I_{x z}(\mathbf{x}) \\
I_{y x}(\mathbf{x}) & I_{y y}(\mathbf{x}) & I_{y z}(\mathbf{x}) \\
I_{z x}(\mathbf{x}) & I_{z y}(\mathbf{x}) & I_{z z}(\mathbf{x})
\end{array}\right]
$$

where the partial derivatives $I . .(\mathbf{x})$ of the volume $I$ are computed after smoothing with an isotropic Gaussian at scale $s$ [22], which corresponds to the expected diameter of the linear structure to be detected.

Let $\left|\lambda_{1}\right| \leq\left|\lambda_{2}\right| \leq\left|\lambda_{3}\right|$ be the eigenvalues of the Hessian $M$. For a line-like object, the smallest eigenvalue $\lambda_{1}$ is small and the corresponding eigenvector

$\mathbf{e}_{1}$ points along the object axis. The other two eigenvalues are larger and of approximately the same magnitude for axially symmetric objects, leading to the following condition for the object voxels:

$$
\left(0 \approx\left|\lambda_{1}\right|\right) \text { and }\left(\left|\lambda_{1}\right| \ll\left|\lambda_{2}\right|\right) \text { and }\left(\lambda_{2} \approx \lambda_{3}\right)
$$

There are various ways to combine the eigenvalues into a single scalar measure [30, 31]. We choose a measure proposed by Frangi et al. [22] and the tubularity measurement is presented as equations below.

$$
\begin{aligned}
J(\mathbf{x}) & =\left(1-\mathrm{e}^{-\frac{\mathcal{R}_{\mathcal{A}}^{2}}{2 \alpha^{2}}}\right)\left(\mathrm{e}^{-\frac{\mathcal{R}_{\mathcal{B}}^{2}}{2 \beta^{2}}}\right)\left(1-\mathrm{e}^{-\frac{\mathcal{S}^{2}}{2 \gamma^{2}}}\right) \\
\text { with } \mathcal{R}_{\mathcal{B}} & =\frac{\left|\lambda_{1}\right|}{\sqrt{\left|\lambda_{2} \lambda_{3}\right|}}, \quad \mathcal{R}_{\mathcal{A}}=\frac{\left|\lambda_{2}\right|}{\left|\lambda_{3}\right|} \\
\mathcal{S} & =\|\mathcal{H}\|_{F}=\sqrt{\sum_{j=1,2,3} \lambda_{j}^{2}}
\end{aligned}
$$

here, $\mathcal{R}_{\mathcal{B}}$ is used to distinguish the structure from a blob-like pattern; $\mathcal{R}_{\mathcal{A}}$ distinguishes between the plate-like and line-like structures; $\mathcal{S}$ quantifies the needle voxels and the background voxels. As recommended by Frangi et al., the $\alpha=\beta=0.5$ are set; the parameter $\gamma$ is tuned on training data as described in [22]. As for a bright object on a dark background $\lambda_{2}$ and $\lambda_{3}$ should be negative, so $J=0$ is set when $\lambda_{2} \geq 0$ or $\lambda_{3} \geq 0$. Some examples in Figures 1,2 and 3 are provided to show a visualize performence of the Frangi's measurement.

\subsection{Oriented filters}

Oriented filters are designed to give a high response for a linear structure with a particular orientation. The orientation is either known or is assumed to be equal to the normalized eigenvector $\mathbf{e}_{1}$ of the Hessian. A standard line filter is $[25]$ :

$$
\zeta(\mathbf{x})=\frac{\partial^{2} I}{\partial \mathbf{e}_{2}^{2}}(\mathbf{x})+\frac{\partial^{2} I}{\partial \mathbf{e}_{3}^{2}}(\mathbf{x}),
$$


where $\mathbf{e}_{2}, \mathbf{e}_{3}$ are the two eigenvectors perpendicular to $\mathbf{e}_{1}$. In our application, the (6) is used to calculate the orient parameter $\zeta(\mathbf{x})$ and further used as a extended feature vector.

Note that second order directional derivatives are easily obtained from the Hessian matrix at a little computational cost

$$
\frac{\partial^{2} I}{\partial \mathbf{v}^{2}}(\mathbf{x})=\mathbf{v}^{\mathrm{T}} \mathcal{H}(\mathbf{x}) \mathbf{v}, \quad \mathbf{v} \in \mathbb{R}^{3},\|\mathbf{v}\|=1
$$

\section{Tool localization with line filtering}

The main improvement over the previous work [20] is that the first two steps now use the shape information and classifiers trained on labeled training data, which significantly improves the localization robustness. It consists of the following four steps:

1. Voxel classification - a set of possible tool voxels $\mathcal{X}_{t}$ are chosen using the intensity of the voxels as well as the Frangi's measure $J(\mathbf{x})(3)$ is selected (Section 3.1).

2. Axis estimation - an approximate position of the tool axis, defined by a polynomial curve with parameters $\mathbf{H}$ is found using RANSAC [21], maximizing the number of inliers ${ }^{1}$ What should be noted here that whether a voxel is an inlier or not is classified using the extent features, for example, oriented filter output $\zeta(\mathbf{x})(6)$ (Section 3.2).

and the respectively.

3. Local optimization - the final solution $\mathbf{H}^{*}$ is found by minimizing the mean squared distance of the inliers identified in the previous step from the tool axis (Section 3.3).

4. Tip localization - the endpoint of the tool is determined by robustly searching for a significant drop in voxel intensity along the axis [14].

\subsection{Voxel classification}

Possible tool voxels $\mathcal{X}_{\mathrm{t}}$ are selected based on a two-dimensional feature vector $\mathbf{m}_{1}(\mathbf{x})=[I(\mathbf{x}) J(\mathbf{x})]$, with voxel intensity $I(\mathbf{x})$ and line filtering output $J(\mathbf{x})$. The following classifiers were tested, see Section 4.2 for experimental comparison.

a) A monolithic linear classifier [33] trained by the Fisher's linear discriminant (FLD), using the feature vector $\mathbf{m}_{1}(\mathbf{x})$ and a pocket algorithm [34] is also implement to keeps the best solution so far.

b) Support Vector Machine (SVM) using kernel function with linear mapping [33], which is composed with the $\mathbf{m}_{1}(\mathbf{x})$ and a randomly chosen subset from the trained data. It is trained using a fast cutting plane algorithm [35].

\footnotetext{
${ }^{1}$ Inliers are points consistent with the model for some set of parameters [32].
} 
c) AdaBoost (adaptive boosting) classifier, which is actually a learning metaalgorithm [36]. Given a labelled training set and a set of weak classifiers, the AdaBoost produces a strong classifier. In our method it is trained with decision stumps [37] and 10 weak classifiers.

d) Waldboost [38] is an algorithm which integrates the AdaBoost training with the Wald's sequential probability ratio test (SPRT). The Waldboost used is trained with domain partitioning weak classifiers [39] with 8 bins. The desired TP rate and FN rate (Table 2) were set to $99 \%$ and $1 \%$, respectively.

e) Cascaded classifier consists of two steps. First, the voxels are thresholded based solely on intensity $I(\mathbf{x})$, the threshold is chosen such that on training data $80 \%$ of true positives are retained. The second step is a linear classifier using both $I(\mathbf{x})$ and $J(\mathbf{x})$. The advantage is that the computationally expensive line filtering can be only performed on a small fraction of the pixels, so the classifier is very fast.

\subsection{Axis estimation}

The tool axis is represented by a polynomial parametric curve

$$
a(t ; \mathbf{H})=\underbrace{\left(\begin{array}{ccc}
h_{11} & \cdots & h_{1 n} \\
h_{21} & \cdots & h_{2 n} \\
h_{31} & \cdots & h_{3 n}
\end{array}\right)}_{\mathbf{H}}\left(\begin{array}{c}
1 \\
t \\
\vdots \\
t^{n-1}
\end{array}\right) ; \quad t \in \mathbb{R}
$$

with $3 n$ parameters $\mathbf{H}$ [20]. We typically use $n=2$ for straight needles.

Robust estimation of the parameters $\mathbf{H}$ is based on RANSAC [21]: in each iteration $n$ points are randomly chosen and the model is fitted [20] by solving the system of $3 n$ linear equations (8). The number of inliers consistent with the estimated model is counted and at the end, the model consistent with the largest number of inliers is reported. The number of iterations is determined automatically, given the allowed probability of missing the optimum [20].

Whether a point (voxel) $\mathrm{x}$ is an inlier is determined by another classifier, using an extended feature vector

$$
\boldsymbol{\omega}=[I(\mathbf{x}) J(\mathbf{x}) d(\mathbf{x} ; \mathbf{H}) \zeta(\mathbf{x} ; \mathbf{H})]
$$

containing the voxel intensity $I$, tubularity $J$, approximate Euclidean distance $d(\mathbf{x} ; \mathbf{H})$ of $\mathbf{x}$ to the axis described by $\mathbf{H}[20]$, and the oriented filter output $\zeta$ (6). Note that both $d$ and $\zeta$ are dependent on the current model, namely for $\zeta$ we use the local axis orientation in the point closest to $\mathbf{x}$. Classifiers (a)-(d) from the previous Section were considered, trained on images with manually determined ground truth.

\subsection{Local optimization}

The final solution $\mathbf{H}^{*}$ is found by minimizing the mean squared distance of the inliers identified in the previous step from the tool axis.

$$
\mathbf{H}^{*}=\arg \min _{\mathbf{H}} \sum_{\mathbf{x} \in \mathcal{X}_{\mathrm{inl}}} d(\mathbf{x} ; \mathbf{H})^{2}
$$




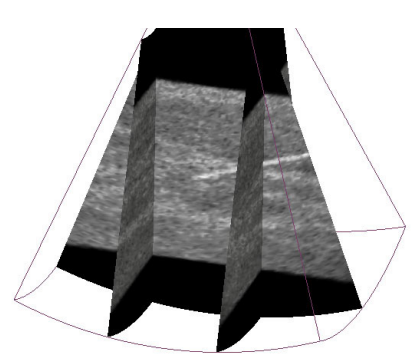

(a)

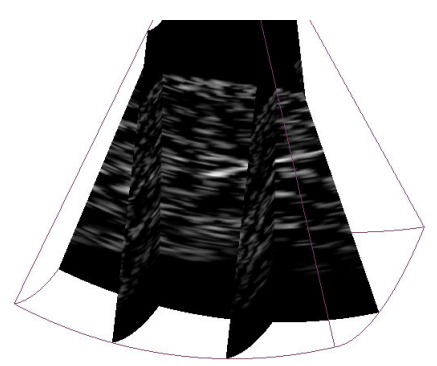

(b)

Figure 1: Example of the line filtering results with straight needle: a) original 3D US image simulated using FIELD II, b) the output of line filtering using Frangi's method.

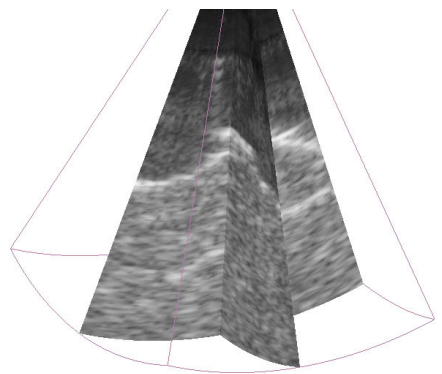

(a)

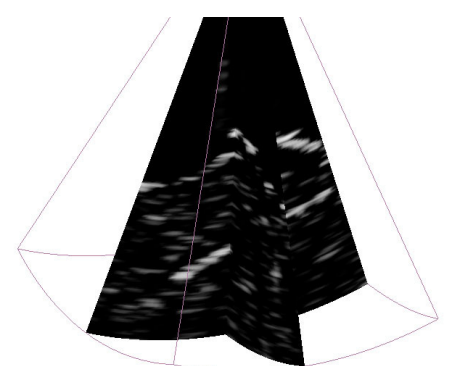

(b)

Figure 2: Example of the line filtering results: a) original 3D US image of the PVA cryogel phantom acquired with Voluson 530D scanner, b) the output of line filtering using Frangi's method.

The optimization is done using a derivative-free Nelder-Mead downhill simplex method [40].

\section{Method evaluation}

\subsection{Visual enhancement}

Despite the fact that Frangi's method provided visually better results, the improvement is evaluated objectively. To evaluate the improvement we have used the contrast ratio $(\mathrm{CR}) \Psi\left(I, \mathcal{X}_{\mathrm{t}}, \mathcal{X}_{\mathrm{bg}}\right)$, defined as the ratio of the mean intensity of the tool voxels $I\left(\mathcal{X}_{\mathrm{t}}\right)$ and the mean intensity of background (non-tool) voxels $I\left(\mathcal{X}_{\mathrm{bg}}\right)$.

$$
\Psi\left(I, \mathcal{X}_{\mathrm{t}}, \mathcal{X}_{\mathrm{bg}}\right)=\frac{\operatorname{mean}\left(I\left(\mathcal{X}_{\mathrm{t}}\right)\right)}{\operatorname{mean}\left(I\left(\mathcal{X}_{\mathrm{bg}}\right)\right)}
$$

The improvement in CR between the original image of intensity $I_{o}$ and filtered image of intensity $I_{f}$ is given by

$$
\Psi^{I m p r}=\frac{\Psi\left(I_{f}, \mathcal{X}_{\mathrm{t}}, \mathcal{X}_{\mathrm{bg}}\right)}{\Psi\left(I_{o}, \mathcal{X}_{\mathrm{t}}, \mathcal{X}_{\mathrm{bg}}\right)}
$$




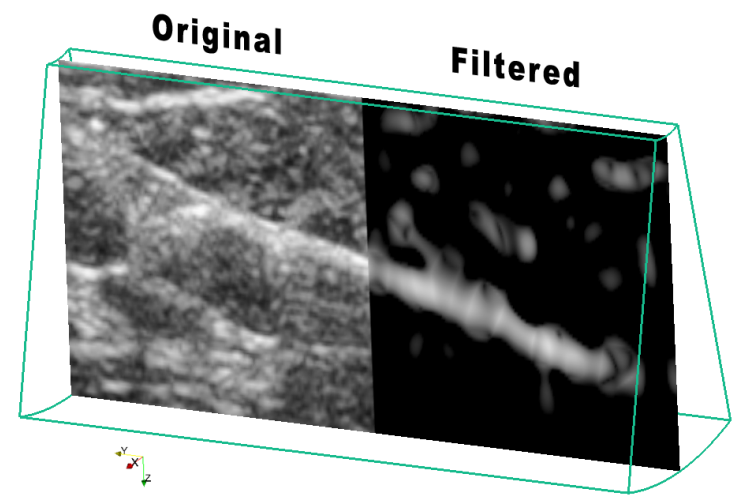

Figure 3: 3D volume of breast biopsy with needle. The left part of the slice contains the original data and the right part contains the data filtered by Frangi's method [22]. The original data were acquired with GE Voluson E8 scanner.

The best improvement in CR is obtained with Frangi's method (Table 7). The computation time is dominated by filtering and calculating the eigenvalues, depends linearly on the number of voxels, and is almost identical for all methods (about 10s for the simulated data using our Matlab implementation [29]).

Table 1: Mean contrast ratio improvement (12) for the three line enhancement methods and three groups of experiments. The best result in each row is set in bold. Numbers in parentheses give the number of volumes in each dataset.

\begin{tabular}{|c||c|c|c|}
\hline Data type (number) & Frangi & Sato & $\mathrm{Li}$ \\
\hline Simul. (28) & $\mathbf{3 . 1 4}$ & 1.96 & 0.65 \\
\hline PVA (8) & $\mathbf{1 6 . 7 8}$ & 4.04 & 3.12 \\
\hline Breast (3) & $\mathbf{4 . 5 4}$ & 2.29 & 1.89 \\
\hline
\end{tabular}

\subsection{Segmentation evaluation}

We compared the ability of classifiers from Section 3.1 to distinguish between tool and background voxels on the simulated data.

The classifiers working points were adjusted so that their specificity was $80 \%$ on the test data

$$
\text { Specificity }=\frac{\mathrm{TN}}{\mathrm{TN}+\mathrm{FP}}
$$

where the symbols are defined in Table 2. Our main performance criterion is precision (also called 'inlier ratio' or positive predictive value, a voxel was considered to be an inlier if it was closer to the tool axis than the tool radius) as it directly influences RANSAC performance. It is defined as

$$
\text { Precision }=\frac{\mathrm{TP}}{\mathrm{TP}+\mathrm{FP}}
$$


Table 2: Confusion matrix illustrates naming conventions for the evaluation of classification results.

\begin{tabular}{|c||c|c|}
\hline & negative predicted & positive predicted \\
\hline \hline actual negative & True Negative (TN) & False Positive (FP) \\
\hline actual positive & False Negative (FN) & True Positive (TP) \\
\hline
\end{tabular}

Table 3: Classifier performance in distinguishing tool voxels. The specificity was fixed to $80 \%$.

\begin{tabular}{|l||c|c|c|}
\hline Type of classifier & Mean precision & Mean sensitivity & Specificity \\
\hline Thresholding & $6.0 \% \pm 2.0 \%$ & $32.7 \% \pm 9.1 \%$ & $80 \%$ \\
\hline Linear (FLD.) & $87.5 \% \pm 1.7 \%$ & $78.8 \% \pm 3.0 \%$ & $80 \%$ \\
\hline SVM & $88.5 \% \pm 1.7 \%$ & $82.1 \% \pm 3.2 \%$ & $80 \%$ \\
\hline AdaBoost & $89.0 \% \pm 2.5 \%$ & $90.0 \% \pm 3.9 \%$ & $80 \%$ \\
\hline WaldBoost & $88.5 \% \pm 1.3 \%$ & $83.2 \% \pm 2.6 \%$ & $80 \%$ \\
\hline Cascade & $97.8 \% \pm 0.9 \%$ & $46.9 \% \pm 8.1 \%$ & $80 \%$ \\
\hline
\end{tabular}

We also evaluate the sensitivity (TP rate)

$$
\text { Sensitivity }=\frac{\mathrm{TP}}{\mathrm{FN}+\mathrm{TP}}
$$

To do the cross validation, 18 simulated datasets have been generated using Field II, with the orientation of needle from $40^{\circ}$ to $110^{\circ}$ with respect to the probe, with a step of $4^{\circ}$. Fifty simulation runs are done on the different pairs of training and testing datasets. For each pair of datasets, 12 out of the 18 are used for training, and the remaining 6 for testing. For each run, the training and testing datasets are randomly chosen from the 18 datasets, without repetition. Table 3 shows the segmentation performance of the different classifiers. The mean precision and sensitivity and their standard deviations are given for specificity $80 \%$. From Table 3, we see that the AdaBoost classifier gives the best results in terms of sensitivity. The cascade classifier has much lower sensitivity but excellent precision, which is important for the subsequent RANSAC step and the speed gain is very important (Table 4). The differences between the sensitivities of the linear, SVM, AdaBoost and WaldBoost classifiers was not found significant by the $t$-test. All classifiers using line filtering perform significantly better than the previous method [20] that uses only intensity (denoted 'Thresholding' in Table 3).

\subsection{Inlier detection}

The inlier detector for RANSAC (Section 3.2) was trained and tested using cross-validation (Section 4.2) on synthetic data taking advantage of the availability of ground truth. The position of the tool was chosen randomly with the angle of the tool with respect to the probe axis $40 \sim 110^{\circ}$ and the distance of the tool from the probe $30 \sim 60 \mathrm{~mm}$. The results for specificity $98 \%$ are reported in Table 5. Best performers were the SVM and WaldBoost. 
Table 4: Time spent on line filtering for different datasets for the line filtering evaluated everywhere ('full') and only for voxels selected by the first step of the cascade classifier ('cascade').

\begin{tabular}{|c|c||r|r|}
\hline Datasets & Size [voxels] & full & cascade \\
\hline \hline Simul. & $53 \times 71 \times 164$ & $10.6 \mathrm{~s}$ & $\mathbf{1 . 0} \mathbf{~ s}$ \\
\hline PVA & $53 \times 71 \times 310$ & $18.8 \mathrm{~s}$ & $\mathbf{2 . 1}$ s \\
\hline Breast tissue & $383 \times 273 \times 208$ & $236.7 \mathrm{~s}$ & $\mathbf{1 4 . 0} \mathbf{~ s}$ \\
\hline
\end{tabular}

Table 5: The performance of the inlier classifier on simulated data.

\begin{tabular}{|l||c|c|c|}
\hline Type of classifier & Precision & Sensitivity & Specificity \\
\hline \hline Linear (FLD) & $94.5 \% \pm 4.4 \%$ & $86.4 \% \pm 4.7 \%$ & $98 \%$ \\
\hline SVM & $99.1 \% \pm 1.9 \%$ & $99.7 \% \pm 1.1 \%$ & $98 \%$ \\
\hline AdaBoost & $84.5 \% \pm 5.6 \%$ & $42.8 \% \pm 4.7 \%$ & $98 \%$ \\
\hline WaldBoost & $97.4 \% \pm 3.1 \%$ & $91.6 \% \pm 8.9 \%$ & $98 \%$ \\
\hline
\end{tabular}

\section{Results on tool localization method with line filtering}

\subsection{Dataset used for method evaluation}

The line filtering methods and proposed localization method were implemented in MATLAB (the MathWorks, Natick, MA) and tested on PC with Intel Core 4 processor at $2.83 \mathrm{GHz}$. The results of experiments are tested on the following datasets.

Simulation. Twenty-eight 3D ultrasound datasets were generated by an ultrasound simulator FIELD II [41, 42] with known ground-truth location of the tool (Figure 1a). Table I gives the parameters used in FIELD II, set to mimic our real ultrasound scanner as closely as possible.

An image of a needle was created by adding a cylinder of diameter $0.6 \mathrm{~mm}$ with highly reflecting scatterers. For each 3D image there were 53 azimuthal planes covering an angle of $40^{\circ}$ and 71 planes covering a lateral angle of $40^{\circ}$; in the axial direction, each pixel corresponds to approximately $0.1 \mathrm{~mm}$.

PVA phantom. Experiments have been done on a PVA cryogel phantom [43] which mimics biological tissue properties.

The PVA cryogel phantom contained an electrode of diameter $0.3 \mathrm{~mm}$. What should be pay attention here is that even the real diameter of the electrode is $0.3 \mathrm{~mm}$, the apparent diameter in the US volume will be almost the same as the simulated one because a procedure of convolution [19] of the PSF. (Figure 2a). Eight 3D ultrasound images of size $53 \times 71 \times 260$ voxels of the PVA cryogel phantom from various positions have been acquired using an ultrasound scanner Voluson 530D with a $7.5 \mathrm{MHz}$ probe. These volumes have different angle pairs $[\alpha, \beta]$ as $\left[20^{\circ}, 90^{\circ}\right],\left[25^{\circ}, 80^{\circ}\right],\left[15^{\circ}, 60^{\circ}\right]$, and $\left[25^{\circ}, 10^{\circ}\right]$, the definition of $\alpha$ and $\beta$ is shown in Figure 4. 
Table 6: Parameters used in FIELD II simulations.

\begin{tabular}{|l||c|}
\hline Parameter name & Value \\
\hline \hline Transducer center frequency $[\mathrm{MHz}]$ & 7.5 \\
\hline Sampling frequency $[\mathrm{MHz}]$ & 27 \\
\hline Speed of sound $[\mathrm{m} / \mathrm{s}]$ & 1540 \\
\hline Elements of the probe & 128 \\
\hline Width of element [mm] & 0.1 \\
\hline Height of element [mm] & 10 \\
\hline Kerf [mm] & 0.017 \\
\hline Focal depth [mm] & 50 \\
\hline Range of scan lines [degree] & {$[-2020]$} \\
\hline Range of scan planes [degree] & {$\left[\begin{array}{ll}-20 & 20\end{array}\right]$} \\
\hline
\end{tabular}

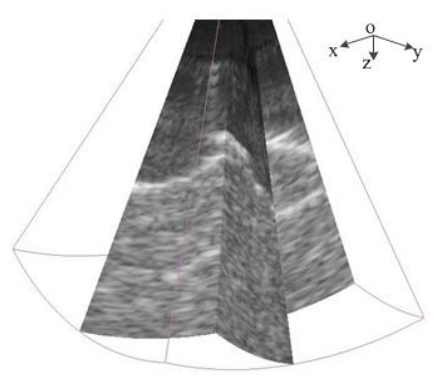

(a) An example of $3 \mathrm{D}$ volume

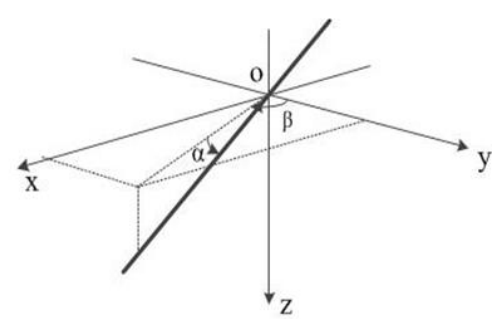

(b) The definition of the two angles of the needle

Figure 4: The definition of the two direction angles of the needle.

Breast biopsy. We tested the method on three real data sets of live breast tissue (Figure 3) with a 11 gauge straight biopsy needle (1 mm outer diameter). 3D ultrasound images were acquired by the GE Voluson E8 scanner with a $12 \mathrm{MHz}$ probe. The size of all volumes was $273 \times 383 \times 208$ voxels.

The needle position for real data sets was determined as an average location given by eight observers. The mean variability for human observers was less than $0.4 \mathrm{~mm}$.

\subsection{Tool localization - testing the complete chain}

The complete proposed localization method was evaluated in terms of the axis accuracy, the failure rate, and the elapsed time. Axis accuracy $\epsilon_{\text {axis }}$ is defined as the maximum Euclidean distance of the true tool endpoints from the estimated tool axis

$$
\epsilon_{\text {axis }}=\max \left\{\left\|E-Q_{1}\right\|,\left\|T-Q_{2}\right\|\right\}
$$

where $E$ is the true intercept point; $T$ is the true tool's tip, and $Q_{1}$ and $Q_{2}$ are the orthogonal projections of $E$ and $T$ on the estimated axis $a(t)$ (see Fig. 5 ). 


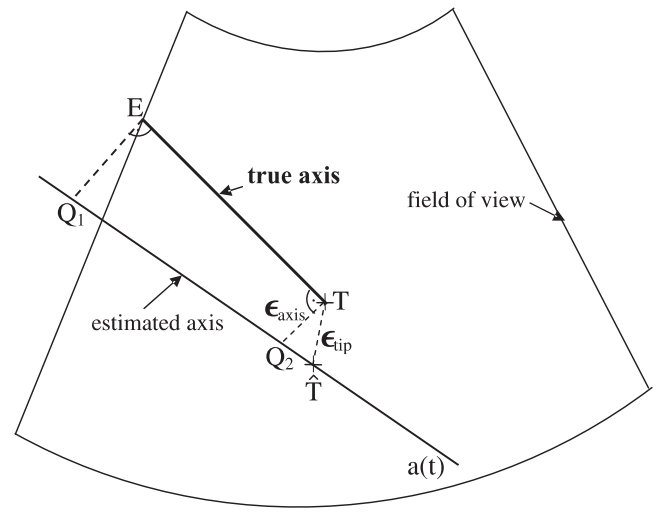

Figure 5: Illustration of the axis accuracy evaluation.The axis accuracy $\epsilon_{\text {axis }}$ is defined as the maximum Euclidean distance between the true endpoints and their projections on the estimated tool axis. The tip accuracy $\epsilon_{\mathrm{tip}}$ is the Euclidean distance between the true tip and the estimate tip.

In [19], it is claimed that for the situation of biopsies, an error of a few $\mathrm{mm}$ can be acceptable. So, the localization was considered to be a failure when the axis accuracy $\epsilon_{\text {axis }}$ was greater than a threshold (set to $3 \mathrm{~mm}$ ) and failures were excluded from accuracy calculations.

We consider six variants of the proposed method: two classifiers for segmentation - (1) a cascaded classifier (denoted CASC) and (2) a linear SVM classifier (SVM); and three inlier classifiers for the axis estimation step - (1) linear SVM (SVM); (2) AdaBoost (ADA); and (3) WaldBoost.

The proposed line filtering method (Section 3.2) was compared to two variants (AxShp and IntDstr) of the previously described RANSAC localization method [20], to the parallel integral projection method (PIP) [14] and its multiresolution variant MR-PIP [17], and the randomized Hough transform (RHT) and its quick variant (Q-RHT) [12]. The reported times include line filtering and pre-processing.

\subsection{Tool localization on the simulated data}

The Contrast Ratio (CR) given by (11) was used to define the image quality. Results (axis accuracy, number of failures, time) for high CR data ( Figure 1a for an example) are reported in Figure 6 . The projection (PIP) methods are the slowest and the Hough transform (RHT) methods have the highest number of failures. Previously described RANSAC based methods [20] are the fastest with a small number of failures. The new method using line-filtering has no failures at all and with the cascade classifier it can be as fast as the earlier methods [20].

Figure 7 shows results for more difficult synthetic data with low CR, see Figure 10 for an example. The methods not using line filtering have failed in all cases. The best performance among line filtering methods was achieved using the SVM+ADA classifier for both stages, the following is the SVM+SVM 

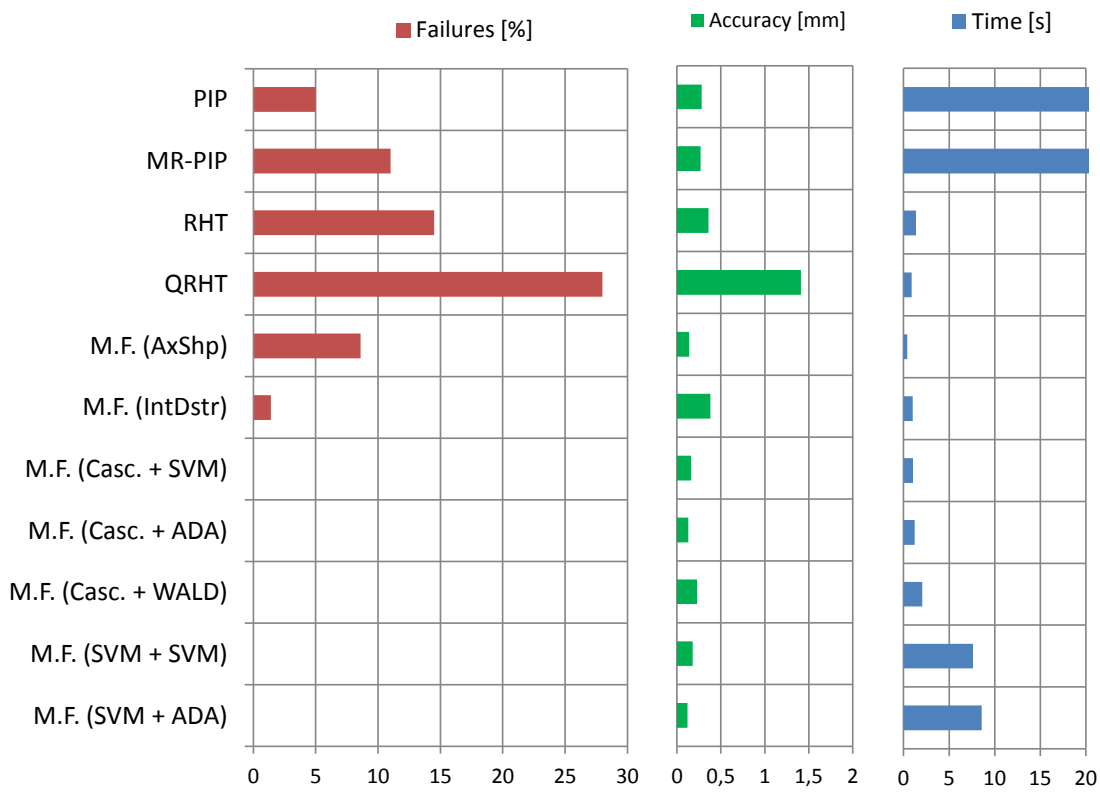

Figure 6: The results of the tool localization on simulated data with high contrast ratio (CR) $(\mathrm{CR}=1.7)$. M.F. stands for model-fitting (RANSAC) methods. The first column is the failure rate for all the methods in percentage; the second column is the axis accuracy in $\mathrm{mm}$; the third column is the time consuming for each method.

classifier. The cascaded classifier decreases the overall time but increases the number of failures.

\subsection{Tool localization on the real data}

The results on real data of the PVA phantom are shown in Figure 8 and example of a localization result with and without line filtering is in Figure 11. All methods not using line filtering fail in $50 \sim 100 \%$ of cases, mostly because of a presence of a highly echogenic $2 \mathrm{D}$ interface. The best performance with respect to the number of failures was achieved by the combination of the Casc+SVM classifier and the SVM+ADA classifiers. The use of cascade classifier in the presegmentation reduces the calculation time, so, considering the aspect of time, the Casc+SVM classifier is better than the SVM+ADA.

Finally, we present results on the three breast biopsy datasets in Figure 9. Methods not using line filtering fail completely. Among line filtering methods, combining SVM and AdaBoost or WaldBoost classifiers works best. Though the combination of two linear SVM classifiers has a more than $50 \%$ failure rate, the accuracy is a little better than the SVM+ADA and the SVM+WALD classifier. 

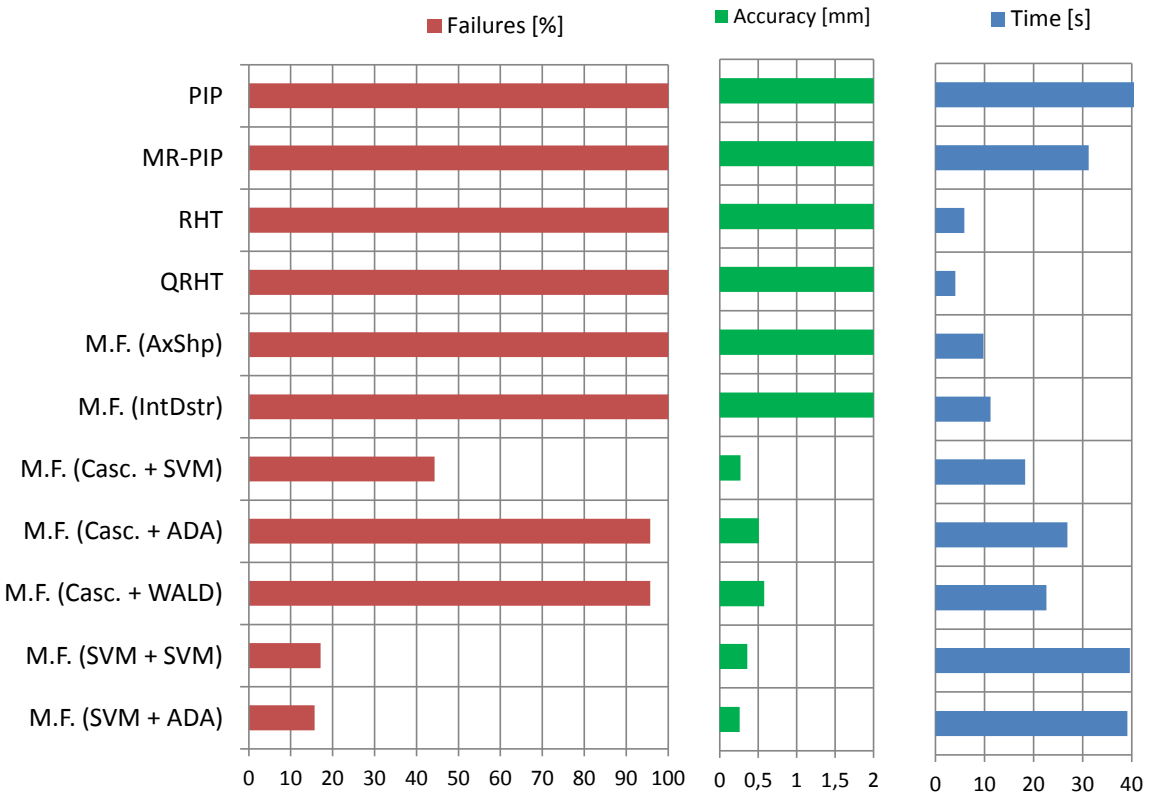

Figure 7: The results of the tool localization on simulated data with low $\mathrm{CR}(\mathrm{CR}=1.1)$. M.F. stands for model-fitting (RANSAC) methods. The first column is the failure rate for all the methods in percentage; the second column is the axis accuracy in $\mathrm{mm}$; the third column is the time consuming for each method.

\section{Discussion}

The line filtering method not only works on the straight needle situation, it also works when there is a $\mathrm{C}$ like deformation of the needle. Figure 12 gives an example of the line filtering result with curved needle. However, we mainly concern on the non-curved situation, so at present, our localization algorithm does not work on the situation of curved needle localization. For sure, to complete our research work, the curved model of RANSAC algorithm will be developped in furture work.

Table 7: Mean contrast ratio improvement (12) for the three line enhancement methods using the simulated volume with a curved needle. The Frangi's method has the best performance.

\begin{tabular}{|c||c|c|c|}
\hline Data type (number) & Frangi & Sato & $\mathrm{Li}$ \\
\hline Simul. curved (4) & $\mathbf{4 . 2 5}$ & 2.97 & 2.71 \\
\hline
\end{tabular}

\section{Conclusions}

We have proposed a new method for tool localization in 3D ultrasound images which exploits its predominantly 1D shape both when initially selecting tool candidate voxels and when evaluating voxels consistent with a particular 

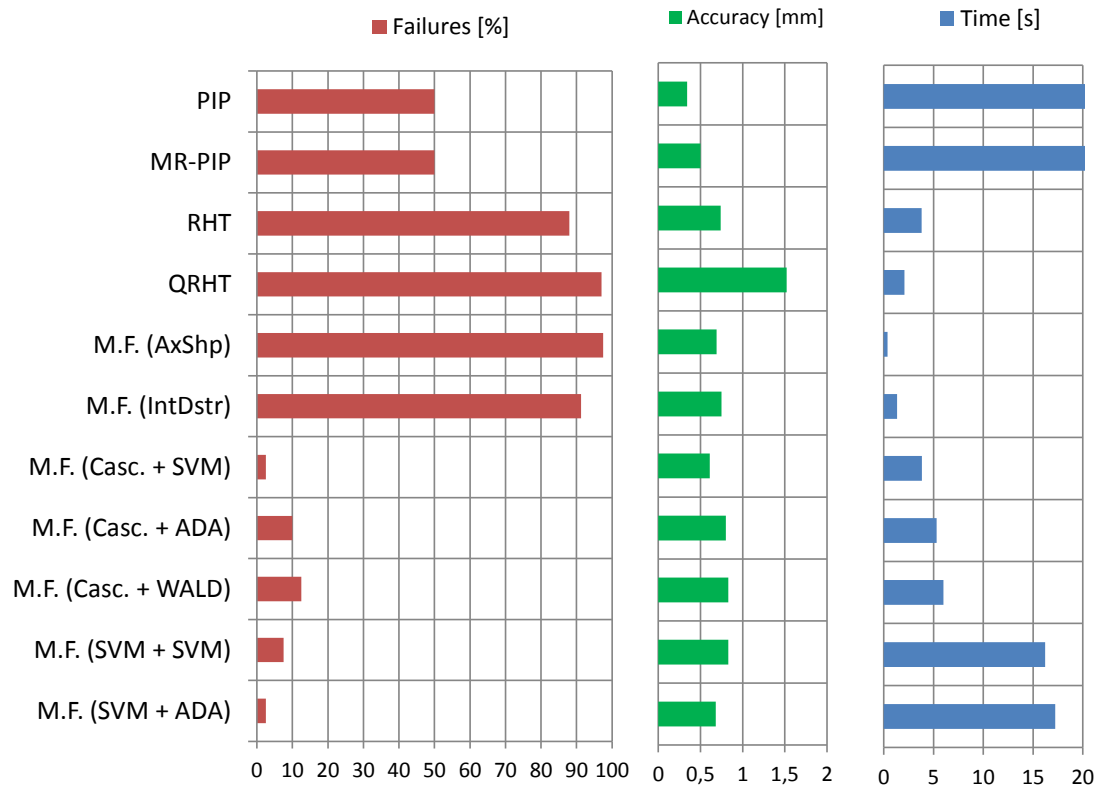

Figure 8: The results of tool localization on the PVA cryogel phantom. The first column is the failure rate for all the methods in percentage; the second column is the axis accuracy in $\mathrm{mm}$; the third column is the time consuming for each method.

tool positions. The robustness is significantly improved with respect to our earlier method [20] as well as with respect to other existing algorithms (Parallel Integral Projection and Randomized Hough Transform).

The best performance in terms of robustness was obtained with SVM classifiers. For datasets with relatively low noise and low clutter, a cascaded classifier approach performs almost as well with much reduced computational complexity.

\section{Acknowledgment}

This work was conducted in the framework of the LabEx CeLyA ("Centre Lyonnais d'Acoustique", ANR-10-LABX-60) and the Czech Science Foundation project P202/11/0111. The 3-D ultrasound breast biopsy and turkey breast data was kindly provided by D. Buckton and C. Perrey from the GE Medical Systems.

[1] N. Abolhassani, R. Patel, M. Moallem, Needle insertion into soft tissue: A survey, Medical Engineering and Physics 29 (4) (2007) 413-431.

[2] A. Abati, A. Simsir, Breast fine needle aspiration biopsy: prevailing recommendations and contemporary practices, Clinics in laboratory medicine 25 (4) (2005) 631-654. 


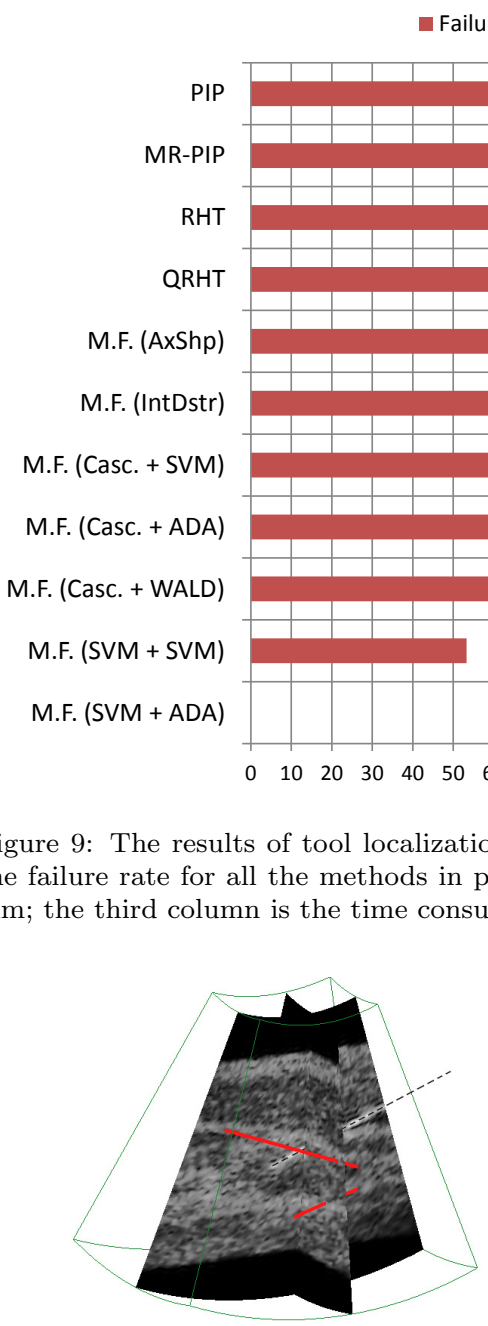

(a)

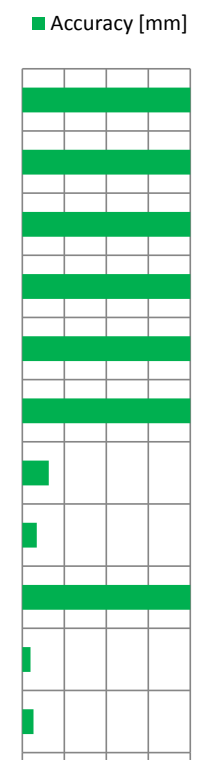

$\begin{array}{lllll}0 & 0,5 & 1 & 1,5 & 2\end{array}$ a Time $[\mathrm{s}]$

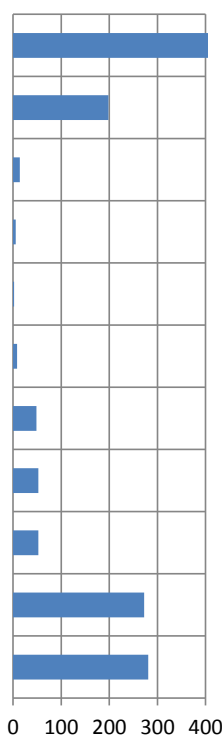

Figure 9: The results of tool localization on real data of breast biopsy. The first column is the failure rate for all the methods in percentage; the second column is the axis accuracy in $\mathrm{mm}$; the third column is the time consuming for each method.

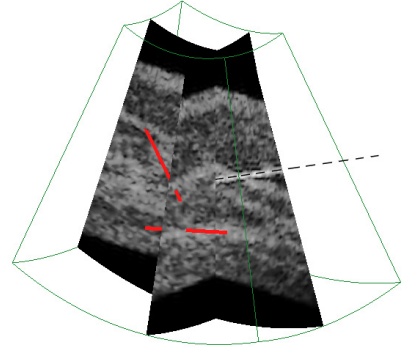

(b)

Figure 10: Visualization of the localization results in simulated 3D ultrasound data with low CR. The dashed line shows the correct position computed using model fitting with line filtering (using SVM classifiers). Two red lines show examples of failures without line filtering (tool model AxShp [20]). Two different views (a) and (b) show the same data set in different orientations.

[3] G. S. Merrick, W. M. Butler, K. E. Wallner, R. W. Galbreath, E. Adamovich, Monotherapeutic brachytherapy for clinically organconfined prostate cancer, West Virginia Medical Journal 101 (4) (2005) $168-171$.

[4] K. Kern, Sentinel lymph node mapping in breast cancer using subareolar 


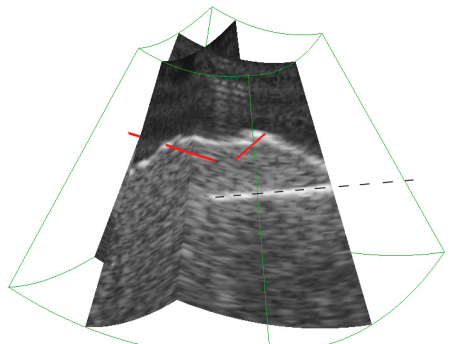

(a)

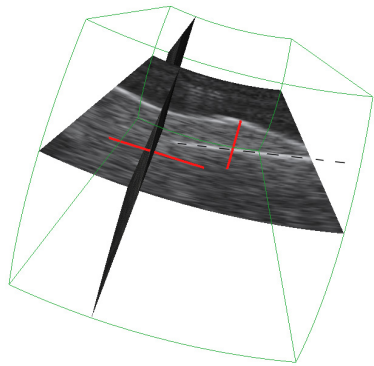

(b)

Figure 11: Visualization of the localization results in 3D ultrasound data of the PVA phantom. The dashed line shows the correct tool position computed using model fitting with line filtering (using SVM classifiers). Two short red lines show examples of failures without line filtering - the localization method was confused by the high intensity layer on the top. Two different views (a) and (b) show the same data set in different orientations.

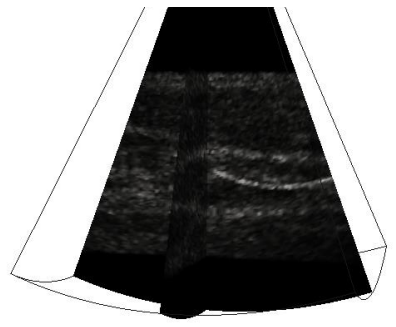

(a)

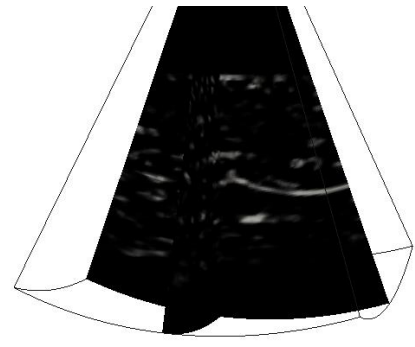

(b)

Figure 12: Example of the line filtering results with curved needle: a) original 3D US image simulated using FIELD II, b) the output of line filtering using Frangi's method.

injection of blue dye, Journal of the American College of Surgeons 189 (6) (1999) 539-545.

[5] R. L. Alterman, D. Sterio, A. Beric, P. J. Kelly, Microelectrode recording during posteroventral pallidotomy, Neurosurgery 44 (2) (1999) 315-321.

[6] V. Horsley, R. H. Clarke, The structure and functions of the cerebellum examined by a new method, Brain 31 (1908) 45-124.

[7] T. M. Peters, Image-guidance for surgical procedures, Physics in Medicine and Biology 51 (14) (2006) R505-R540.

[8] J. W. Cannon, J. A. Stoll, I. S. Salgo, Real time 3-dimensional ultrasound for guiding surgical tasks, Computer aided surgery 8 (2003) 82-90.

[9] S. H. Okazawa, R. Ebrahimi, J. Chuang, R. N. Rohling, S. E. Salcudean, Methods for segmenting curved needles in ultrasound images, Medical image analysis 10 (3) (2006) 330-342. 
[10] M. Ding, Z. Wei, L. Gardi, D. B. Downey, A. Fenster, Needle and seed segmentation in intra-operative 3D ultrasound-guided prostate brachytherapy, Ultrasonics 44 (1) (2006) 331-336.

[11] M. Aboofazeli, P. Abolmaesumi, P. Mousavi, G. Fichtinger, A new scheme for curved needle segmentation in three-dimensional ultrasound images, in: 6thIEEE International Symposium on Biomedical Imaging: From Nano to Macro (ISBI), 2009, pp. 1067-1070.

[12] H. Zhou, W. Qiu, M. Ding, S. Zhang, Automatic needle segmentation in 3D ultrasound images using 3D improved Hough transform, Proc. SPIE Med. Imag. Parallel Process Images, Optim. Techn.(MIPPR).

[13] H. R. S. Neshat, R. V. Patel, Real-time parametric curved needle segmentation in 3D ultrasound images, in: 2nd IEEE RAS \& EMBS International Conference on Biomedical Robotics and Biomechatronics, IEEE press, 2008, pp. 670-675.

[14] M. Barva, M. Uhercik, J.-M. Mari, J. Kybic, J.-R. Duhamel, H. Liebgott, V. Hlavác, C. Cachard, Parallel integral projection transform for straight electrode localization in 3-d ultrasound images, IEEE Transactions on Ultrasonics, Ferroelectrics and Frequency Control 55 (7) (2008) 1559-1569.

[15] S. Helgason, The Radon Transform, 2nd Edition, Birkhauser Boston, New York, USA, 1999.

[16] W. Qiu, M. Ding, M. Yuchi, Needle segmentation using 3D quick randomized Hough transform, in: First International Conference on Intelligent Networks and Intelligent Systems, ICINIS'08, 2008, pp. 449-452.

[17] M. Uhercik, J. Kybic, H. Liebgott, C. Cachard, Multi-resolution parallel integral projection for fast localization of a straight electrode in 3D ultrasound images, in: 5th IEEE International Symposium on Biomedical Imaging: From Nano to Macro (ISBI), IEEE, 2008, pp. 33-36.

[18] P. M. Novotny, J. A. Stoll, N. V. Vasilyev, P. J. del Nido, P. E. Dupont, R. D. Howe, GPU based real-time instrument tracking with three dimensional ultrasound, Medical image analysis 11 (5) (2007) 458-64.

[19] J. M. Mari, C. Cachard, Ultrasonic scanning of straight micro tools in soft biological tissues: Methodology and implementation, Ultrasonics 51 (5) (2011) 632-638.

[20] M. Uherčík, J. Kybic, H. Liebgott, C. Cachard, Model Fitting Using RANSAC for Surgical Tool Localization in 3-D Ultrasound Images, IEEE Transactions on Biomedical Engineering (BME) 57 (8) (2010) 1907-1916.

[21] M. A. Fischler, R. C. Bolles, Random sample consensus: A paradigm for model fitting with applications to image analysis and automated cartography, Communications of the ACM 24 (6) (1981) 381-395. 
[22] A. F. Frangi, W. J. Niessen, K. L. Vincken, M. A. Viergever, Multiscale vessel enhancement filtering, in: Medical Image Computing and ComputerAssisted Interventation-MICCAI'98, Springer, 1998, pp. 130-137.

[23] S. Chaudhuri, S. Chatterjee, N. Katz, M. Nelson, M. Goldbaum, Detection of blood vessels in retinal images using two-dimensional matched filters, IEEE Transactions on medical imaging 8 (3) (1989) 263-269.

[24] A. D. Hoover, V. Kouznetsova, M. Goldbaum, Locating blood vessels in retinal images by piecewise threshold probing of a matched filter response, IEEE Trans. on Medical Imaging 19 (3) (2000) 203-210.

[25] W. T. Freeman, E. H. Adelson, The design and use of steerable filters, IEEE Transactions on Pattern analysis and machine intelligence 13 (9) (1991) 891-906.

[26] G. González, F. Aguet, F. Fleuret, M. Unser, P. Fua, Steerable Features for Statistical 3D Dendrite Detection, in: Proceedings of MICCAI, SpringerVerlag, 2009, pp. 625-632.

[27] S. Aylward, E. Bullitt, S. Pizer, D. Eberly, Intensity ridge and widths for tubular object segmentation and description, in: Proceedings of the Workshop on Mathematical Methods in Biomedical Image Analysis, IEEE, 1996, pp. 131-138.

[28] J. Staal, M. D. Abràmoff, M. Niemeijer, M. A. Viergever, B. van Ginneken, Ridge-based vessel segmentation in color images of the retina, IEEE Transactions on Medical Imaging 23 (4) (2004) 501-509.

[29] M. Uherčík, Surgical tools localization in 3D ultrasound images, Research report, INSA de Lyon, Prague, Czech Republic (April 2011).

[30] Q. Li, S. Sone, K. Doi, Selective enhancement filters for nodules, vessels, and airway walls in two- and three-dimensional CT scans, Medical Physics 30 (8) (2003) 2040-2051.

[31] Y. Sato, S. Nakajima, N. Shiraga, H. Atsumi, S. Yoshida, T. Koller, G. Gerig, R. Kikinis, Three-dimensional multi-scale line filter for segmentation and visualization of curvilinear structures in medical images, Medical image analysis 2 (2) (1998) 143-168.

[32] M. Barva, Localization of Surgical Instruments in 3D Ultrasound Images, PhD Thesis CTU-CMP-2007-12, Center for Machine Perception, K13133 FEE Czech Technical University, Prague, Czech Republic (June 2007).

[33] R. O. Duda, P. E. Hart, D. G. Stork, Pattern Classification (2nd Edition), Wiley-Interscience, 2000.

[34] S. I. Gallant, Perceptron-based learning algorithms, IEEE Trans. on Neural Networks 1 (2) (1990) 179-191. 
[35] V. Franc, S. Sonnenburg, OCAS optimized cutting plane algorithm for support vector machines, in: Proceedings of ICML, 2008, pp. 320-327.

[36] Y. Freund, R. E. Schapire, A Decision-Theoretic Generalization of On-Line Learning and an Application to Boosting, Journal of Computer and System Sciences 55 (1997) 119-139.

[37] T. Svoboda, J. Kybic, V. Hlaváč, Image Processing, Analysis \& and Machine Vision-A MATLAB Companion, 1st Edition, Thomson, Toronto, Canada, 2007.

[38] J. Sochman, J. Matas, Waldboost-learning for time constrained sequential detection, in: IEEE Computer Society Conference on Computer Vision and Pattern Recognition, CVPR 2005., Vol. 2, IEEE Computer Society, Washington, DC, USA, 2005, pp. 150-156.

[39] R. E. Schapire, Y. Singer, Improved boosting algorithms using confidencerated predictions, Machine Learning 37 (1999) 297-336.

[40] J. A. Nelder, R. Mead, A simplex method for function minimization, The Computer Journal 7 (1965) 308-313.

[41] J. A. Jensen, Field: A Program for Simulating Ultrasound Systems, in: 10TH Nordicbaltic Conference On Biomedical Imaging, Vol. 34, Springer, New York, 1996, pp. 351-353.

[42] J. A. Jensen, N. B. Svendsen, Calculation of pressure fields from arbitrarily shaped, apodized, and excited ultrasound transducers, IEEE Transactions on Ultrasonics, Ferroelectrics and Frequency Control 39 (2) (1992) 262-267.

[43] F. Duboeuf, A. Basarab, H. Liebgott, E. Brusseau, P. Delachartre, D. Vray, Investigation of PVA cryogel Young's modulus stability with time, controlled by a simple reliable technique, Medical Physics 36 (2) (2009) 656661. 